\title{
Pathophysiology of acute kidney injury in severe acute pancreatitis-an overview
}

\begin{abstract}
Acute pancreatitis (AP) is a common disorder of the pancreas and its severity ranges from mild self-limited disease to severe acute pancreatitis (SAP). Acute kidney injury (AKI) is a frequent complication of severe acute pancreatitis and carries a very poor prognosis, particularly if renal replacement therapy (RRT) is required, with mortality rates between $25 \%$ and $75 \%$. Different key pathophysiologic processes include release of pancreatic enzymes with resulting impairment of renal microcirculation, hypoxemia, hypovolemia, intra abdominal hypertension, endotoxin and cytokines mediated injury.
\end{abstract}

Keywords: acute pancreatitis, acute kidney injury, renal replacement therapy, intra abdominal hypertension, endotoxin
Volume 10 Issue $4-2019$

\section{Piyush Mathur,' Sandeep Vaishnav²}

'Consultant Nephrologist, Santokba Durlabhji Memorial

Hospital, India

${ }^{2}$ Consultant Gastroenterologist, Santokba Durlabhji Memorial

Hospital, India

Correspondence: Piyush Mathur, DNB (Internal Medicine), DNB (Nephrology), Consultant Nephrologist, Santokba Durlabhji Memorial Hospital, Jaipur, India, Tel +91 7023333189, Email piyushnephro@gmail.com

Received: June 27, 2019 | Published: August 23, 2019
Abbreviations: AP, acute pancreatitis; SAP, severe acute pancreatitis; AKI, acute kidney injury; PAF, platelet activating factor; SIRS, systemic inflammatory reaction syndrome; NO, nitric oxide; AA, arachodonic acid; ACS, abdominal compartment syndrome; IAP, intra-abdominal pressure; $\mathrm{RBC}$, red blood cell

\section{Introduction}

Acute pancreatitis (AP) is a common disorder of the pancreas and its severity ranges from mild self-limited disease to severe acute pancreatitis (SAP). Occurrence of acute pancreatitis is increasing with incidence rates ranging from 20 to 80 per 100,000 per annum and varying across countries. ${ }^{1}$ SAP is an acute clinical syndrome characterized by acute onset, rapid progression, high incidence of complications with failure of multiple organs and high mortality, estimated as from $7 \%$ to $47 \% .^{2-4}$ Acute kidney injury (AKI) is a frequent complication of severe acute pancreatitis and carries a very poor prognosis, particularly if renal replacement therapy (RRT) is required, with mortality rates between $25 \%$ and $75 \%{ }^{5}$ The exact mechanism of AKI in patients with AP is complex and not very well understood. Different key pathophysiologic processes include release of pancreatic enzymes with resulting impairment of renal microcirculation, hypoxemia, hypovolemia, intra abdominal hypertension, endotoxin and cytokines mediated injury. The aim of this article is to summarize current concepts in the pathophysiology of renal injury in SAP.

\section{Pathophysiology of AKI in acute pancreatitis}

The key pathophysiologic process involves release of pancreatic enzymes from inflamed pancreas leading to autodigestion of pancreas and triggering cascade of events contributing to AKI. The activated enzymes and proteases released into circulation causes endothelial damage leading to extravasation of fluids from vascular space. This leads to intravascular volume depletion, hypotension and third spacing of fluid with increased intra abdominal pressure. All these events ultimately lead to decrease renal perfusion, renal vasoconstriction, and hypercoagubility and fibrin deposition in glomeruli. ${ }^{6}$

\section{Role of pancreatic enzymes and toxic substances}

Conventionally, hypovolemia and hypotension are considered as constant factors contributing to AKI in AP. Recent studies have shown that various toxic substances released from activation of kallikrein kinin system are involved in pathogenesis of AKI. These substances include trypsin, chymotrypsin, bradykinin, histamine, phospholipases, prostaglandins $\mathrm{E}$ and various other endotoxins. ${ }^{7}$ Vasoactive peptides released during activation of kallikrein kinin system increases glomerular permeability and causes injury to renal tubules and interstitium. Phospholipases released decompose the phospholipids and lecithin of the cell membrane. Lipid metabolites, such as free fatty acids, acylcarnitine, acyl-coenzyme etc are all membrane-active factors that can destroy the cell membrane. The activated complement system can produce $\mathrm{C} 5 \mathrm{~b}$ and $\mathrm{C} 5 \mathrm{~b}$ and combine $\mathrm{C} 6, \mathrm{C} 7, \mathrm{C} 8$ and $\mathrm{C} 9$ to generate a "membrane attack complex" that destroys the cell membrane and results in irreversible cell injury. ${ }^{8}$ Animal studies done earlier have shown that histamine from pancreatic exudate of dogs with experimental acute pancreatitis and injection of ascitic fluid from experimental dogs with acute pancreatitis caused increased vascular permeability, hypovolemia, and hypotension. ${ }^{7,9}$

But, recent study by Dawara et al. ${ }^{10}$ concluded that trypsin activity is not necessary for caerulein-induced pancreatitis. Trypsinogen activation is definitely not the only factor that determines the severity of pancreatitis and development of AKI. Studies have shown that other pathways including activation of NF- $\mathrm{kB}$ pathway, ${ }^{11}$ elevated Ras signaling ${ }^{12}$ are also able to induce pancreatitis. The notion that trypsin is sufficient but not required for the development of pancreatitis might be able to explain the failed clinical trials with trypsin inhibitors.

Thus, although hypotension and hypovolemia may be the initial culprits in causing AKI early during acute pancreatitis, toxic substances in the pancreatic exudate may subsequently contribute to AKI. 


\section{Role of inflammatory mediators}

Recent studies haves found that some inflammatory mediators play important roles in SAP complicated with multiple organ injury. The main inflammatory mediators implicated in SAP complicated by AKI are cytokines (TNF $\alpha$, IL 6, and TGF), phospholipase A2 (PLA2), platelet activating factor (PAF), nuclear factor kappa $\beta(\mathrm{NF} \kappa \beta)$ and endotoxins.

Cytokines are released in AP; their concentration constantly increases during evolvement of AP and may contribute to the pathogenesis of AKI. ${ }^{13}$

In AP activated neutrophilic granulocyte releases various cytokines of which TNF $\alpha$ is an important inflammatory mediator for onset and progression of SAP and AKI. ${ }^{14}$ TNF $\alpha$ acts directly on pancreatic duct cells, glomeruli and renal tubules causing inflammation, ischemia and necrosis of both pancreatic duct cells and renal tubular epithelium. Moreover, excessive TNF $\alpha$ enters the blood circulation, further stimulates neutrophillic granulocytes promoting their aggregation. These over activated neutrophillic granulocytes then releases interleukins: IL-1b, IL-8 IL-6, and platelet activating factor (PAF) causing a cytokine cascade reaction that promotes the systemic inflammatory reaction syndrome (SIRS) and aggravates pancreatic and renal injury. ${ }^{15,16}$

IL-1b can interact with TNF- $\alpha$ to induce or aggravate organ injury. It also has chemotaxis and activating effects on granulocyte and can stimulate the production of other inflammatory mediators, such as IL8, IL-6. IL-1 and IL-6 can act on endothelial cells, causing them to lower their thrombomodulin activity, aggravate renal ischemia, form thrombus and activate inflammatory cells to release Nitric Oxide (NO) and oxygen free radicals to directly cause renal injury. IL 8 can activate and induce $\mathrm{T}$ and $\mathrm{B}$ cell differentiation, enhance NK cells for killing target cells, promote phagocytosis and play an important role in tissue injury mediated by neutrophilic granulocytes. ${ }^{17}$ Studies have shown that during SAP the levels of IL-6 and IL-8 always increase concurrently and that these positively correlate with the state of SAP. ${ }^{18,19}$

TNF- $\alpha$ also increases level of PAF which further promotes granulocyte aggregation, aggravates inflammatory reactions, increases capillary permeability and aggravates renal tubule injury. ${ }^{20}$

Oxygen free radicals have been known to play an important role in the pathogenesis of pancreatitis of some experimental models. ${ }^{21}$ Oxygen free radicals are involved in initiation of pancreatitis and are important mediators of tissue damage. ${ }^{22,23}$ Oxygen free radicals may react with proteins and enzymes, leading to lipid peroxidation of the cell and organellar membranes, protein denaturation and increased capillary permeability, ischemia, and direct kidney cell membrane injury. ${ }^{23}$

Nitric oxide (NO) is recognized as an important mediator of several physiological and pathophysiological processes. In the pancreas a growing amount of evidence has been presented that NO exerts various effects in the pathophysiology of acute pancreatitis. ${ }^{24,25}$ NO mediates renal injury by interacting with oxygen free radicals and causes direct toxic effect on renal tubules. NO also lowers the reaction of the blood vessel to stagnated substances and causes renal ischemia. ${ }^{19}$

Phospholipase A2 (PLA2), which is one of major body lipases, is widely distributed in the plasma and is secreted mainly by the neutrophilic granulocytes and macrophages in the pancreatic acinar cells. Level of PLA2 is consistent with severity ${ }^{26}$ and prognosis of $\mathrm{SAP}^{27}$ and also plays key role in pathogenesis of AKI. ${ }^{28}$ In SAP; large quantity of PLA2 is released in blood from activated neutrophilic granulocytes and macrophages. This PLA2 decomposes membrane phospholipids thereby disintegrating cell membrane resulting in the massive leakage of lysosome enzyme out of the cell, but it also generates bioactive free fatty acids and soluble lecithin to destroy the function and structure of the systemic cell and organ system. In kidneys, PLA2 hydrolyze the renal tubular epithelial cell membrane lecithin, leading to the generation of free fatty acid and hemolytic lecithin. This hemolytic lecithin can dissolve the renal tubular epithelial cell membrane. PLA2 also increases vascular permeability and generation of Arachodonic acid (AA), which produces thromboxane (TXA2). TXA2 is a potent capillary vasoconstrictor substance and platelet aggregation promoter that is able to induce platelet deformation, release and secretion, formation of micro thrombus, vascular occlusion and other pathological changes that can result in the abnormal vasomotor function in renal microcirculation, decline in renal blood flow and tissue perfusion. These changes can cause serious injury to the kidney resulting in AKI. ${ }^{29,30}$

In recent years, many experimental studies have implicated nuclear factor-kappa $\mathrm{B}(\mathrm{NF}-\kappa \mathrm{B})$ activation as an early and central event in the progression of inflammation in $\mathrm{AP}^{31,32} \mathrm{NF \kappa} \beta$ is a nuclear transcription factor responsible for regulating the transcription of a wide variety of genes involved in immunity and inflammation and links the initial acinar injury to systemic inflammation and perpetuate the inflammation. NF- $\mathrm{kB}$ activation increases markedly as severity of pancreatitis increases. This abnormal activation NFк $\beta$ promotes the genetic transcription of various pro-inflammatory factors like TNF- $\alpha$, IL-1, IL-6. All these inflammatory mediators can further activate $N F \kappa \beta$ thus propagating the inflammatory reactions. $N F \kappa \beta$ has ability to bind on intracellular adhesion molecule 1(ICAM 1) and due to this bonding in renal tissue, ICAM 1 mediates the adhesion between the polymorphonuclear granulocyte (PMN) and vascular endothelial cells, playing an important role in the aggregation process of PMN with tissue. The aggregated inflammatory cells in glomeruli can induce direct toxic effects that cause cell morphological changes, proliferation, capillary injury and the formation of crescents. ${ }^{19}$

\section{Abdominal compartment syndrome}

Abdominal compartment syndrome (ACS) is a severe complication of SAP and is because of increased intra-abdominal contents by ileus, ascites, intra-abdominal bleeding and most importantly interstitial fluid accumulation due to increased capillary permeability due to endothelial damage. ${ }^{33}$ The increased intra-abdominal pressure (IAP) $>20 \mathrm{~mm} \mathrm{Hg}$ reduces the blood supply to the abdominal organs, leading to low perfusion in the kidneys causing AKI. ${ }^{34}$ The mechanism of intra-abdominal hypertension induced AKI is not clear, but intraabdominal hypertension may compress and compromise the kidney blood flow in both the arterial and venous vasculature, leading to decreased perfusion pressure, increased venous pressure, decreased venous blood flow, and increased kidney parenchymal pressure. This results in decreased glomerular filtration pressure, and impaired micro-vascular function and oxygen delivery, and precipitates ischemic kidney injury. ${ }^{6,35}$

\section{Hemodynamic alterations}

In SAP, blood viscosity and red blood cell (RBC) aggregation index increases markedly which disturbs the microcirculation in pancreas and other organs. This leads to decline in renal microcirculation 
blood flow, an increase in renal vascular resistance. Increased kidney vascular resistance was demonstrated in dogs with experimental acute pancreatitis. ${ }^{36}$ The whole blood viscosity and afferent vessel contraction here may directly cause an increase in glomeruli resistance to lower renal blood flow. Increased viscosity and RBC rigidity affect the microcirculation of the renal tubule capillary and cause renal cortex and medulla microcirculation disturbance, followed by renal failure.

\section{Conclusion}

In conclusion, in addition to hypovolemia, SAP complicated with renal injury is caused by manifold factors and understanding of the complex pathophysiology of both diseases, AKI and SAP, is required to determine adequate subsequent diagnostic and therapeutic procedure.

\section{Acknowledgments}

None.

\section{Conflicts of interest}

The authors declare that there are no conflicts of interest.

\section{Funding}

None.

\section{References}

1. Afghani E, Pandol SJ, Shimosegawa T, et al. Acute Pancreatitis-Progress and Challenges: A Report on an International Symposium. Pancreas. 2015;44(8):1195-210.

2. Kes P, Vucicevic Z, Ratkovic-Gusic I, et al. Acute renal failure complicating severe acute pancreatitis. Ren Fail. 1996;18(4):621-628.

3. Kumar R, Pahwa N, Jain N. Acute kidney injury in severe acute pancreatitis: an experience from a tertiary care center. Saudi J Kidney Dis Transpl. 2015;26(1):56-60.

4. Lin HY, Lai JI, Lai YC, et al. Acute renal failure in severe pancreatitis: A population-based study. Ups J Med Sci. 2011;116(2):155-159.

5. Pupelis G. Renal failure in acute pancreatitis. Timing of dialysis and surgery. Przegl Lek. 2000;57 Suppl 5:29-31.

6. Nassar TI, Qunibi WY. AKI Associated with Acute Pancreatitis. Clin J Am Soc Nephrol. 2019;4(7):1106-1115.

7. Satake K, Kanazawa G, Hiura A, et al. Renal function in experimentally induced acute pancreatitis in dogs: how it is affected by the nephrotoxic substance in pancreatic exudate from ascitic fluid. Jpn J Surg. 1991;21(1):88-95.

8. Hietaranta A, Kemppainen E, Puolakkainen P, et al. Extracellular phospholipases A2 in relation to systemic inflammatory response syndrome (SIRS) and systemic complications in severe acute pancreatitis. Pancreas. 1999;18(4):385-91.

9. Ofstad E, Amundsen E, Hagen PO. Experimental acute pancreatitis in dogs. II. Histamine release induced by pancreatic exudate. Scand J Gastroenterol. 1969;4(1):75-79.

10. Dawra R, Sah RP, Dudeja V, et al. Intra-acinar trypsinogen activation mediates early stages of pancreatic injury but not inflammation in mice with acute pancreatitis. Gastroenterology. 2011;141(6):2210-2217.e2.

11. Chen X, Ji B, Han B, et al. NF-kappaB activation in pancreas induces pancreatic and systemic inflammatory response. Gastroenterology.
2002;122(2):448-457.

12. Ji B, Tsou L, Wang H, et al. Ras activity levels control the development of pancreatic diseases. Gastroenterology. 2009;137(3):1072-1082.

13. Lipsett PA. Serum cytokines, proteins, and receptors in acute pancreatitis: mediators, markers, or more of the same? Critical Care Med. 2001;29(8):1642-1644.

14. Ogawa M. Acute pancreatitis and cytokines: "second attack" by septic complication leads to organ failure. Pancreas. 1998;16(3):312-325.

15. Mayer J, Rau B, Gansauge F, et al. Inflammatory mediators in human acute pancreatitis: clinical and pathophysiological implications. Gut. 2000;47(4):546-452.

16. Fisic E, Poropat G, Bilic-Zulle L, et al. The Role of IL-6, 8, and 10, sTNFr, CRP, and Pancreatic Elastase in the Prediction of Systemic Complications in Patients with Acute Pancreatitis. Gastroenterol Res Pract. 2013;2013:282645.

17. Gross V, Andreesen R, Leser HG, et al. Interleukin-8 and neutrophil activation in acute pancreatitis. Eur J Clin Invest. 1992;22(3):200-203.

18. Wereszczynska-Siemiatkowska U, Dabrowski A, Siemiatkowski A, et al. Serum profiles of E-selectin, interleukin-10, and interleukin-6 and oxidative stress parameters in patients with acute pancreatitis and nonpancreatic acute abdominal pain. Pancreas. 2003;26(2):144-152.

19. Zhang XP, Wang L, Zhou YF. The pathogenic mechanism of severe acute pancreatitis complicated with renal injury: a review of current knowledge. Dig Dis Sci. 2008;53(2):297-306.

20. Emanuelli G, Montrucchio G, Dughera L, et al. Role of platelet activating factor in acute pancreatitis induced by lipopolysaccharides in rabbits. Eur J Pharmacol. 1994;261(3):265-272.

21. Sanfey H, Bulkley GB, Cameron JL. The pathogenesis of acute pancreatitis. The source and role of oxygen-derived free radicals in three different experimental models. Ann Surg. 1985;201(5):633-639.

22. Schoenberg MH, Buchler M, Gaspar M, et al. Oxygen free radicals in acute pancreatitis of the rat. Gut. 1990;31(10):1138-1143.

23. Rau B, Poch B, Gansauge F, et al. Pathophysiologic role of oxygen free radicals in acute pancreatitis: initiating event or mediator of tissue damage? Ann Surg. 2000;231(3):352-360.

24. Satoh A, Shimosegawa T, Abe T, et al. Role of nitric oxide in the pancreatic blood flow response to caerulein. Pancreas. 1994;9(5):574579 .

25. Rahman SH, Ammori BJ, Larvin $\mathrm{M}$, et al. Increased nitric oxide excretion in patients with severe acute pancreatitis: evidence of an endotoxin mediated inflammatory response? Gut. 2003;52(2):270-274.

26. Mirkovic D. The role of phospholipase A2 in the pathogenesis of respiratory damage in hemorrhagic necrotizing pancreatitis--assessment of a new experimental model. Vojnosanit Pregl. 2000;57(6):625-633.

27. Aufenanger J, Samman M, Quintel M, et al. Pancreatic phospholipase A2 activity in acute pancreatitis: a prognostic marker for early identification of patients at risk. Clin Chem Lab Med. 2002;40(3):293-297.

28. Nevalainen TJ, Hietaranta AJ, Gronroos JM. Phospholipase A2 in acute pancreatitis: new biochemical and pathological aspects. Hepatogastroenterology. 1999;46(29):2731-2735.

29. Iida T, Yokoi H, Kawarada Y. The effects of a thromboxane A2 synthesis inhibitor and a prostaglandin I2 analogue on experimental acute necrotizing pancreatitis in rats. Pancreas. 1998;17(2):140-147.

30. Dumnicka P, Maduzia D, Ceranowicz P, et al. The Interplay between Inflammation, Coagulation and Endothelial Injury in the Early Phase of Acute Pancreatitis: Clinical Implications. Int J Mol Sci. 2017;18(2). 
31. Suk K, Yeou Kim S, Kim H. Regulation of IL-18 production by IFN gamma and PGE2 in mouse microglial cells: involvement of NF-kB pathway in the regulatory processes. Immunol Lett. 2001;77(2):79-85.

32. Izumi T, Saito Y, Kishimoto I, et al. Blockade of the natriuretic peptide receptor guanylyl cyclase-A inhibits NF-kappaB activation and alleviates myocardial ischemia/reperfusion injury. J Clin Invest. 2001;108(2):203-213.

33. Papavramidis TS, Marinis AD, Pliakos I, et al. Abdominal compartment syndrome - Intra-abdominal hypertension: Defining, diagnosing, and managing. J Emerg Trauma Shock. 2011;4(2):279-291.

34. Li H, Qian Z, Liu Z, et al. Risk factors and outcome of acute renal failure in patients with severe acute pancreatitis. J Crit Care. 2010;25(2):225229.
35. Patel DM, Connor MJJ. Intra-Abdominal Hypertension and Abdominal Compartment Syndrome: An Underappreciated Cause of Acute Kidney Injury. Adv Chronic Kidney Dis. 2016;23(3):160-166.

36. Nishiwaki H, Ko I, Hiura A, et al. Renal microcirculation in experimental acute pancreatitis of dogs. Ren Fail. 1993;15(1):27-31. 